\title{
THEORETICAL MODELLING OF THE INFRARED FLUORESCENCE BY INTERSTELLAR POLYCYCLIC AROMATIC HYDROCARBONS
}

\author{
W. A. SCHUTTE, A. G. G. M. TIELENS, and L. J. ALLAMANDOLA \\ Space Science Division, MS:245-6, NASAAmes Research Center, Moffett \\ Field, CA 94035, U.S.A.
}

\begin{abstract}
We modelled the IR emission of interstellar PAHs. Substantial differences between the IR properties of interstellar and laboratory PAHs are found, possibly resulting from ionization. The various IR features being dominated by distinctly different size PAHs, their observed relative intensities are sensitive indicators of the size distribution. A number of applications of our model related to future ISO and SIRTF IR data are pointed out.
\end{abstract}

\section{Introduction}

The interstellar emission features at $3.3,6.2,7.7,8.6,11.3$, and $12.7 \mu \mathrm{m}$ have been ascribed to fluorescence by interstellar Polycyclic Aromatic Hydrocarbons (PAHs). We investigated the relation between the PAH size distribution and the observed emission spectrum by theoretical modelling. We furthermore studied to what extend interstellar PAHs may be dehydrogenated.

\section{The Model}

We defined the PAH size distribution by extrapolating the MRN power law distribution for visual grains down to a minimum size of $24 \mathrm{C}$ atoms. To calculate the fluorescence we used the thermal approximation (Léger and Puget 1984). The results differ by less than $10 \%$ from exact quantum-mechanical calculations.

\section{Results}

Figure 1 shows how various sizes of PAHs contribute to the emission in the mid-IR features and the far-IR $25 \mu \mathrm{m}$ IRAS band. The various interstellar emission bands are dominated by PAHs of distinctly different sizes, from $\leq 80 \mathrm{C}$ atoms for the $3.3 \mu \mathrm{m}$ feature, to $\sim 10^{3} \mathrm{C}$ atoms for the far-IR emission. The relative intensities of the various mid-IR bands and the far-IR emission are therefore sensitive indicators of the size distribution.

Using cross sections of PAHs measured in the laboratory for the mid-IR features to model the observed emission results in a considerable underestimate of the 6.2 and 7.7 $\mu \mathrm{m} \mathrm{C}-\mathrm{C}$ stretching and $8.6 \mu \mathrm{m} \mathrm{C}-\mathrm{H}$ bending features relative to the 11.3 and $12.7 \mu \mathrm{m}$ $\mathrm{C}-\mathrm{H}$ out-of-plane bending modes. Although dehydrogenating the PAHs by $\sim 95 \%$ reproduces the correct $7.7 / 11.3 \mu \mathrm{m}$ ratio (de Muizon et al. 1990), this solution faces a number of problems. First, it results in a much too weak $8.6 \mu \mathrm{m}$ feature. Second, the observed $12.7 \mu \mathrm{m}$ feature which is likely due to the coupled out-of-plane modes of 2 
adjacent $\mathrm{C}-\mathrm{H}$ groups, is not reproduced, since only isolated $\mathrm{C}-\mathrm{H}$ groups can survive at this severe dehydrogenation. Alternatively, we can solve the discrepancy using fully hydrogenated PAHs by increasing the intrinsic strengths of the 7.7 and $8.6 \mu \mathrm{m}$ features by a factor of 6 and the $6.2 \mu \mathrm{m}$ feature by a factor of 2.4. Such an increase could possibly be related to the interstellar PAHs being ionized (deFrees et al. 1991).

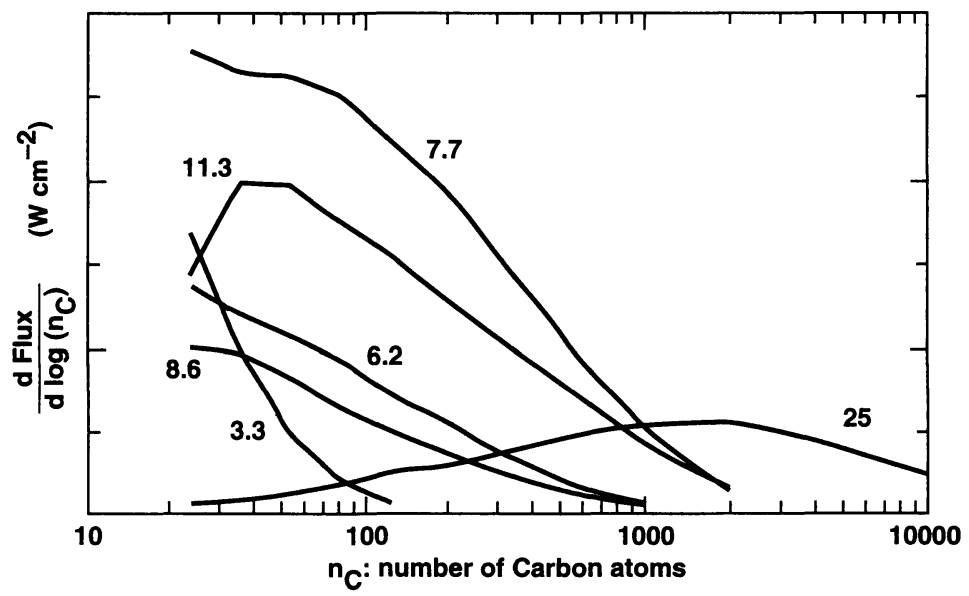

Figure 1. Distribution over the $P A H$ size distribution of the emission in the mid-IR features and the IRAS $25 \mu \mathrm{m}$ band.

\section{Future Apllications}

High sensitivity, single aperture observations of the entire emission spectrum from 3 $\mu \mathrm{m}$ to far-IR wavelengths as will likely be obtained by the ISO and SIRTF satellites, together with theoretical modelling of the PAH emission may answer a number of important questions. First, how does the size distribution vary from object to object ? Second, are there characteristic emission features in the far-IR $(\lambda>15 \mu \mathrm{m})$ that could be used to determine the abundance of individual PAHs ? Next, is there a feature around $14 \mu \mathrm{m}$ due to the out-of-plane bending mode of 3 adjacent $\mathrm{C}-\mathrm{H}$ groups and, if so, what do the relative intensities of the 3 out-of-plane modes reveal about the molecular structure and degree of hydrogenation ? And, finally, how do the properties change for "young" PAHs in planetary nebula and "old" PAHs in reflection nebulae and cirrus clouds?

\section{References}

deFrees, D. J., Miller, M. D., Talbi, D., Pauzat, F., and Ellinger, E. (1991) 'Theoretical IR spectra of PAHs and the effect of ionization', preprint.

Jourdain de Muizon, M. J., d'Hendecourt, L. B., and Geballe, T. R. (1990) 'Polycyclic aromatic hydrocarbons in the near-infrared spectra of 24 IRAS sources', A\&A 227, 526-541.

Léger, A., and Puget, J. L. (1984) 'Identification of the "unidentified IR emission features of interstellar dust ?', A\&A 137, L5. 